\title{
A Simple and Effective Method to Generate Lentiviral Vectors for Ex Vivo Gene Delivery to Mature Human Peripheral Blood Lymphocytes
}

\author{
Shicheng Yang,, ${ }^{1 *}$ Neel K. Karne,,2* Stephanie L. Goff,,3 Mary A. Black, Hui Xu, Daniela Bischof, ${ }^{1,}$ \\ Kenneth Cornetta, ${ }^{4-6}$ Steven A. Rosenberg, Richard A. Morgan, and Steven A. Feldman ${ }^{1}$
}

\begin{abstract}
Human ex vivo gene therapy protocols have been used successfully to treat a variety of genetic disorders, infectious diseases, and cancer. Murine oncoretroviruses (specifically, gammaretroviruses) have served as the primary gene delivery vehicles for these trials. However, in some cases, such vectors have been associated with insertional mutagenesis. As a result, alternative vector platforms such as lentiviral vectors (LVVs) are being developed. LVVs may provide advantages compared with gammaretroviral vectors, including the ability to transduce large numbers of nondividing cells, resistance to gene silencing, and a potentially safer integration profile. The aim of this study was to develop a simplified process for the rapid production of clinical-grade LVVs. To that end, we used a self-inactivating bicistronic LVV encoding an MART (melanoma antigen recognized by $\mathrm{T}$ cells)-1-reactive $\mathrm{T}$ cell receptor containing oPRE, an optimized and truncated version of woodchuck hepatitis virus posttranslational regulatory element (wPRE). Using our simplified clinical production process, 293 cells were transiently transfected in roller bottles. The LVV supernatant was collected, treated with Benzonase, and clarified by modified step filtration. LVV produced in this manner exhibited titers and a biosafety profile similar to those of cGMP (current Good Manufacturing Practices) LVVs previously manufactured at the Indiana University Vector Production Facility in support of a phase I/II clinical trial. We describe a simple, efficient, and low-cost method for the production of clinical-grade LVV for ex vivo gene therapy protocols.
\end{abstract}

\section{Introduction}

G ENE THERAPY, in particular, adoptive cell transfer (ACT) is a promising strategy for the treatment of a variety of genetic disorders, infectious diseases, and cancer (Blaese et al., 1995; Walter et al., 1995; Heslop et al., 1996; Cavazzana-Calvo et al., 2000; Gaspar et al., 2004; Morgan et al., 2006). For ex vivo gene therapy protocols, peripheral blood lymphocytes (PBLs) can be genetically modified to express a tumor-reactive $\mathrm{T}$ cell receptor (TCR) or chimeric antigen receptor (CAR) directed against antigens including, but not limited to, MART (melanoma antigen recognized by $\mathrm{T}$ cells)-1, gp100, carcinoembryonic antigen (CEA), NY-ESO-1, CD19, or other tumor antigens. In initial phase I/II clinical trials with limited numbers of patients treated, response rates ranged between 13 and 100\% (Morgan et al., 2006; Kochenderfer et al., 2010; Kalos et al., 2011; Parkhurst et al., 2011; Porter et al., 2011; Robbins et al., 2011). Importantly, genetically modified PBLs can mediate tumor regression and can be redirected against a variety of cancers on the basis of the availability of tumorspecific TCRs or CARs.

To date, gammaretroviral vectors have been used primarily for the genetic modification of a variety of cells ex vivo because the availability of stable packaging cell lines easily allows for large-scale manufacture. However, with the use of vectors that mediate the stable integration of the transgene

\footnotetext{
*Both S.Y. and N.K.K. contributed equally to this manuscript.

†Present address: Division of Neurosurgery, Department of Surgery, Duke University Medical Center, Durham, NC 27710.

${ }^{1}$ Surgery Branch, National Cancer Institute, National Institutes of Health, Bethesda, MD 20892.

${ }^{2}$ Department of Surgery/General Surgery, SUNY Upstate Medical University, Syracuse, NY 13210.

${ }^{3}$ Department of Surgery, College of Physicicans and Surgeons, Columbia University, New York, NY 10032.

Departments of ${ }^{4}$ Medical and Molecular Genetics, ${ }^{5}$ Microbiology and Immunology, and ${ }^{6}$ Medicine, Indiana University School of Medicine, Indianapolis, IN 46202.
} 
into the host genome, there is an increased risk of insertional mutagenesis that can result in altered gene expression profiles. This phenomenon was observed during a clinical trial wherein $\mathrm{CD} 34^{+}$stem cells were transduced with a gammaretroviral vector in order to correct $X$-linked severe combined immunodeficiency disease (X-SCID) in children, resulting in the malignant transformation of cells from the transduced population (Cavazzana-Calvo et al., 2000; Hacein-Bey-Abina et al., 2003). No such adverse events were associated with an adenosine deaminase (ADA)-SCID trial carried out at about the same time (Blaese et al., 1995; Aiuti et al., 2002). These trials suggest that insertional mutagenesis associated with gammaretroviral transduction of $\mathrm{CD} 34^{+}$ stem cells can occur, as in the case of the X-SCID trials; however, further effort will be required to determine whether the genotoxicity is vector dependent, transgene dependent, or both (Woods et al., 2006; Shaw and Kohn, 2011).

At present, there has been one report of malignant transformation associated with lentiviral vector (LVV) transduction of $\mathrm{CD}^{+}{ }^{+}$stem cells (Cavazzana-Calvo et al., 2010). However, the number of clinical trials involving LVV transduction of human $\mathrm{CD} 4^{+}$stem cells or other immune cells, for that matter, is small and more time will be needed to assess the degree and/or risk of insertional mutagenesis associated with LVV. On the basis of current data, it appears that LVVs have a more "random" integration pattern and do not target the enhancers located at the $5^{\prime}$ end of transcriptionally active genes (Wu et al., 2003). In addition, LVVs are able to transduce large numbers of minimally activated immune cells, making them an attractive gene delivery vehicle for ex vivo gene therapy trials (Cavalieri et al., 2003).

To support multiple small phase I/II ACT clinical trials, such as those conducted at the Surgery Branch of the National Cancer Institute (NCI, Bethesda, MD) or other translational research institutions, a simple and cost-effective LVV production platform is essential. At present, the cGMP (current Good Manufacturing Practices) standard for LVV production involves transient vector production followed by concentration, purification, and diafiltration via tangential flow filtration and/or column chromatography (for a review, see Segura et al., 2011). These processes are costly, require significant process development efforts in order to minimize product loss, and may not be required for phase I/II clinical trials. We describe a simplified production method that can be adapted to most clinical research environments and can easily support phase I/II clinical trials, each of which may require production of a unique clinical-grade LVV.

\section{Materials and Methods}

\section{Patient peripheral blood mononuclear cells} and cell lines

Melanoma cell lines 526 and 624 (HLA-A*0201/MART- ${ }^{+}$) and 888 and 938 (HLA-A2 ${ }^{-}$) were isolated from surgically resected metastases as previously described (Topalian et al., 1989) and were cultured in R10 medium consisting of RPMI 1640 (Life Technologies, Carlsbad, CA) containing $10 \%$ fetal bovine serum (FBS; HyClone, Logan, UT). All peripheral blood mononuclear cells and lymphocytes used for transduction and as feeder cells were obtained from aphereses of Surgery Branch, NCI patients according to institutional review board-approved protocols and cultured in
AIM-V medium (Life Technologies) supplemented with $5 \%$ human AB serum (Valley Biomedical, Winchester, VA), 2-mercaptoethanol, nonessential amino acids, HEPES, and L-glutamine (Life Technologies). 293T cells were cultured in D10 medium containing Dulbecco's modified Eagle's medium (DMEM; Life Technologies) with 10\% FBS. All cells were cultured at $37^{\circ} \mathrm{C}$ in a $5 \% \mathrm{CO}_{2}$ humidified incubator.

\section{Vector construction}

The lentiviral vector (LVV) constructs used were derived from pRRL-cPPT-MSCVU3-GFP-Wsin (MSCV-GFP) encoding the green fluorescent protein (GFP) gene driven by the murine stem cell virus (MSCV) U3 promoter (Jones et al., 2009). The detailed pedigree of the lentiviral vector used herein is as follows. Gene transfer vector pRRL-PGK-GFPWsin, a gift from D. Trono (Ecole Polytechnique Fédérale de Lausanne [EPFL], Lausanne, Switzerland), is a modification of pRRL originally described by L. Naldini and colleagues (Dull et al., 1998). pRRL-PGK-GFP-Wsin was modified by Lizée and colleagues to include the human immunodeficiency virus-1 (HIV-1) central polypurine tract (cPPT) to yield the vector pRRL-cPPT-PGK-GFP-Wsin (Lizée et al., 2003). The phosphoglycerate kinase (PGK) promoter from this vector was replaced with the U3 promoter region of the murine stem cell virus (MSCV), resulting in the vector pRRLcPPT-MSCVU3-GFP-Wsin (Jones et al., 2009). In addition, the woodchuck hepatitis virus posttranslational response element (W or wPRE) was replaced with an optimized and truncated version ( $\mathrm{O}$ or oPRE) from which the $\mathrm{X}$ protein open reading frame (ORF) and promoter were deleted (Schambach et al., 2006). The oPRE sequence, flanked with SalI and EcoRI restriction enzyme sites, was synthesized (Epoch Biolabs, Missouri City, TX) and cloned into corresponding sites of pRRL-cPPT-MSCVU3-GFP-Wsin. This vector was designated pRRL-cPPT-MSCVU3-GFP-Osin. In addition, a lentiviral vector encoding the DMF5 TCR $\alpha$ and $\beta$ chains targeting the melanoma antigen recognized by $\mathrm{T}$ cells, MART-1, was constructed as previously described (Johnson et al., 2006; Yang et al., 2008a,b). The $\alpha$ and $\beta$ chains of DMF5 TCR were linked with a furin cleavage site (RAKR) followed by an SGSG spacer and F2A peptide; the consolidated sequence was codon optimized and engineered with $A s c \mathrm{I}$ and SalI sites by GeneArt (Life Technologies), and cloned into the corresponding sites of vectors pRRL-cPPT-MSCVU3-GFPWsin and -Osin. These vectors were designated pRRL-cPPTMSCVU3-coDMF5-Wsin and -Osin. All the constructs were confirmed by restriction enzyme digestion and sequencing.

\section{Lentivirus production}

For small-scale vector preparations, transient lentiviral vector was produced as previously described (Yang et al., 2008b). For lentiviral vector produced at the Indiana University Vector Production Facility (IUVPF), the day before transfection $20 \times 5$-stack cell factories (Nunc, Rochester, NY) were seeded with $2 \times 10^{8} 293 \mathrm{~T}$ cells in $500 \mathrm{ml}$ of D10. On the day of transfection, the culture medium was replaced with $500 \mathrm{ml}$ of D10. Vesicular stomatitis virus glycoprotein (VSV-G)pseudotyped lentiviral vector was produced transiently by transfection, using calcium phosphate (Promega, Madison, WI) and a four-plasmid system. Each cell factory received 
plasmid DNA (555 $\mu \mathrm{g}$ of transfer vector, $192.5 \mu \mathrm{g}$ of pMDG.1, $277.5 \mu \mathrm{g}$ of $\mathrm{pMDLg} / \mathrm{pRRE}$, and $137.5 \mu \mathrm{g}$ of pRSV-REV). The next day, the medium was exchanged with harvest medium consisting of $500 \mathrm{ml}$ of OptiPRO (Life Technologies) plus $5 \mathrm{mM}$ sodium butyrate and the supernatant (10 liters) was harvested $24 \mathrm{hr}$ later and held at $4^{\circ} \mathrm{C}$. The cell factories were refed with $500 \mathrm{ml}$ of harvest medium and the supernatant (10 liters) was harvested a second time, $24 \mathrm{hr}$ later, after which both harvests were pooled (20-liter final volume), clarified by step filtration, and concentrated by tangential flow filtration (Spectrum, New Brunswick, NJ) down to 2 liters. The concentrated vector was treated with Benzonase (50 U/ml; EMD Chemicals, San Diego, CA) for $1 \mathrm{hr}$ at room temperature followed by diafiltration with 5 volumes (10 liters) of AIM-V medium (Life Technologies). The final product was aliquoted into 50-ml Cryocyte bags (Baxter Healthcare, Deerfield, IL) and stored at $-80^{\circ} \mathrm{C}$ until further use. For lentiviral vector produced at the Surgery Branch Vector Production Facility (SBVPF), the day before transfection, $850-\mathrm{cm}^{2}$ roller bottles (Corning, Acton, MA) were seeded with $6.5 \times 10^{7} 293 \mathrm{~T}$ cells in $100 \mathrm{ml}$ of D10. On the day of transfection, the culture medium was exchanged with $100 \mathrm{ml}$ of D10. VSV-G-pseudotyped lentiviral vector was produced as described previously, using the following plasmid DNA concentrations per bottle: $108 \mu \mathrm{g}$ of transfer vector, $38 \mu \mathrm{g}$ of pMDG.1, $70 \mu \mathrm{g}$ of $\mathrm{pMDLg} / \mathrm{pRRE}$, and $27 \mu \mathrm{g}$ of pRSV-REV. Vector supernatants were harvested at 48 and $72 \mathrm{hr}$, pooled, treated with Benzonase for $1 \mathrm{hr}$ at room temperature, and subjected to clarification by modified step filtration as described (Feldman et al., 2011). The final product was aliquoted and stored at $80^{\circ} \mathrm{C}$ until further use. Additional Benzonase treatment conditions are described in Supplementary Table S1 (supplementary data are available online at http://www.liebertpub.com/hgtb). Supernatant containing viral vectors was titered by transduction of PBLs as described subsequently or with a p24 kit (Cell Biolabs, San Diego, CA). Residual Benzonase was detected by ELISA according to the manufacturer's protocol (EMD Chemicals). Residual lentiviral packaging plasmid DNA was detected by real-time PCR as described previously (Sastry et al., 2004). A standard curve was generated with plasmid pMDLg/pRRE, ranging from 1.0 to $0.0000001 \mathrm{ng}$.

\section{$P B L$ transduction and vector titration}

On day 0 , PBLs $\left(5 \times 10^{6} \mathrm{cell} / \mathrm{ml}, 2 \mathrm{ml} /\right.$ well $)$ were stimulated with interleukin (IL)-2 (300 IU/ml) and anti-CD3/ anti-CD28 beads (ratio of beads to cells was 3:1; Life Technologies) in tissue culture-treated 6-well plates (Becton Dickinson, Franklin Lakes, NJ) and incubated overnight at $37^{\circ} \mathrm{C}$ and $5 \% \mathrm{CO}_{2}$. Serial dilutions of vector supernatant containing protamine sulfate $(10 \mu \mathrm{g} / \mathrm{ml})$ were applied to $1 \times 10^{6}$ stimulated PBLs (final volume, $1 \mathrm{ml}$ ) in individual wells of a 24-well plate (Becton Dickinson). The plates were centrifuged at $2000 \times g$ for $2 \mathrm{hr}$ at $32^{\circ} \mathrm{C}$ followed by incubation at $37^{\circ} \mathrm{C}$ and $5 \% \mathrm{CO}_{2}$. The next day, the medium was replaced with AIM-V medium containing 5\% human serum (as described previously) and IL-2 (300 IU / $\mathrm{ml})$, and the cells were transferred to $25-\mathrm{cm}^{2}$ tissue culture flasks (Sartstedt, Newton, NC) at a density of $0.3 \times 10^{6}$ cells $/ \mathrm{ml}$. Vector titers were calculated as follows: [(\% tetramer-positive cells $\times$ total cell number $\times$ dilution factor)] $\div$ supernatant volume.

\section{Fluorescence-activated cell-sorting analysis}

Receptor expression was analyzed with phycoerythrin (PE)-conjugated HLA-A*0201/MART-1:27-35L peptide tetramer (Beckman Coulter, San Jose, CA) in combination with allophycocyanin (APC)-, PE-cyanine 7 (Cy7)-, and APCCy7-conjugated antibodies directed against human CD3, CD4, and/or CD8 (BD Biosciences, San Jose, CA), respectively. Isotype controls were in accordance with the manufacturer's recommendations. Immunofluorescence analyzed as relative log fluorescence of live cells was measured with a FACSCanto II flow cytometer (BD Biosciences). Cells were stained in fluorescence-activated cell-sorting (FACS) buffer containing phosphate-buffered saline (PBS) and $0.5 \%$ bovine serum albumin.

\section{Cytokine release assays}

TCR-transduced effector cells $\left(1 \times 10^{5}\right)$ and melanoma cell lines $\left(1 \times 10^{5}\right)$ were placed in overnight coculture $(200 \mu \mathrm{l})$ at $37^{\circ} \mathrm{C}$ and $5 \% \mathrm{CO}_{2}$. Supernatants were harvested for ELISA to detect interferon (IFN)- $\gamma$ (Thermo Scientific, Waltham, MA).

\section{Real-time PCR}

Briefly, real-time PCR was used to detect residual lentiviral packaging plasmid DNA (VSV-G) as described previously (Sastry et al., 2004). Detection of adenovirus E1a and simian virus 40 (SV40) DNAs was conducted at the IUVPF by quantitative PCR (qPCR) on PBL genomic DNA 14 days posttransduction with LVV. The detection limits for both adenovirus E1a and SV40 qPCR assays were $\geq 10$ copies per $0.2 \mu \mathrm{g}$ of DNA.

\section{Replication-competent lentivirus}

The replication-competent lentivirus (RCL) assay was performed at the IUVPF according to U.S. Food and Drug Administration (FDA) guidelines. Briefly, in order to amplify any potential RCL, C8166-45 cells were inoculated with the test article and cultured for a minimum of 21 days. The indicator phase is carried out by inoculating naive C8166-45 cells with cell-free medium from the amplification phase and passaging the cells for an additional 7 days. At the end of the indicator phase, supernatants and genomic DNA are collected and assayed for p24 and $\psi$-gag sequences, respectively. The presence of RCL in the test sample is indicated by detection of $\psi$-gag sequences by qPCR in the indicator cell DNA and p24 antigen in the indicator cell supernatants. Controls were run in triplicate. The positive control was HIV-1 diluted to 5 infectious units and the negative control was mock-infected medium. For detection of p24, the p24 standard curve ranged from 12.5 to $100 \mathrm{pg}$ of p24 per milliliter. For the assay to be valid, all the negative controls had to be negative or below the limits of detection and at least one of the three replicates in the positive control samples had to be positive. All RCL assays conducted for this study were valid.

\section{Statistical analysis}

Results of cytokine secretion were compared by paired Student $t$ test. The $p$ values are two-tailed and indicated in the figures. Results of TCR modifications were compared by one-way analysis of variance (ANOVA). Values are presented as the mean \pm standard error of the mean (SEM). 


\section{Results}

\section{Development of a clinical lentiviral vector}

To enhance both titer and transgene expression, we developed a self-inactivating lentiviral vector (LVV) containing an internal MSCV promoter driving expression of our gene of interest (GOI) along with the woodchuck hepatitis virus posttranscriptional regulatory element (W or wPRE), pRRLcPPT-MSCVU3-GOI-Wsin (Fig. 1A) (Yang et al., 2008b). The wPRE has been reported to contain an open reading frame encoding the $X$ protein, which has the potential to be oncogenic (Fig. 1B) (Schambach et al., 2005). We replaced the wPRE with a codon-optimized and truncated version of the posttranscriptional regulatory element (oPRE) in which the $X$ protein open reading frame promoter has been deleted along with any ATG start sites in ORFs encoding sequences greater than 25 amino acids in length (Fig. 1B). When PBLs were transduced with GFP-encoding vectors, the titers of pRRL-cPPTMSCVU3-GFP-Wsin (wPRE-GFP) and pRRL-cPPT-MSCVU3GFP-Osin (oPRE-GFP) were not significantly different $(4.75$ $[ \pm 1.2] \times 10^{6}$ and $4.34[ \pm 1.2] \times 10^{6} \mathrm{TU} / \mathrm{ml}$, respectively; Fig. $\left.2 \mathrm{~A}\right)$. Inclusion of oPRE in the pRRL lentiviral backbone did not affect the stability of the transgene, as the level of GFP expression was maintained for up to 30 days for both constructs (Fig. 2B). For subsequent evaluations, we compared three lentiviral constructs encoding an anti-MART-1 (DMF5) TCR and wPRE (wDMF5) or oPRE (oDMF5) and a codon-optimized version of the DMF5 TCR in conjunction with oPRE (coDMF5; Fig. 1A). There was no significant difference in titer between any of the constructs tested (wDMF5, $8.3[ \pm 0.1] \times 10^{6} ;$ oDMF5, $7.7[ \pm 1.3] \times 10^{6} ;$ coDMF5, 7.8 $[ \pm 1.6] \times 10^{6}$, respectively). The coDMF5 TCR was selected for clinical use on the basis of a significant increase in functional activity as measured by IFN- $\gamma$ secretion when compared with the other vectors (Supplementary Fig. S1).

\section{Effect of Benzonase on DNA removal and PBL transduction}

Because of increased demand for lentiviral vectors encoding a variety of tumor antigen-specific TCRs or CARs for cancer immunotherapy clinical trials, a simple and efficient production platform would be beneficial. To that end, we evaluated our lentiviral vector production process, using flatstock cell culture vessels and a simplified downstream process consisting of Benzonase treatment followed by clarification by modified step filtration. The final vector product was aliquoted without additional concentration and/or diafiltration and stored at $-80^{\circ} \mathrm{C}$ until further use. On the basis of previous ACT clinical trials, in order to achieve a patient cell dose of $1-3 \times 10^{10}$ lymphocytes, using an LVV, one would need to transduce $5 \times 10^{8}$ PBLs, assuming a 50-fold cell expansion after 2 weeks in culture (Yang et al., 2008b), which would require approximately $100 \mathrm{ml}$ of unconcentrated LVV supernatant. Thus, a 100-ml volume of LVV supernatant could be considered a single patient dose. The current World Health Organization (WHO) regulatory guidance limit for residual DNA in biological products derived from continuous cell lines is $10 \mathrm{ng} /$ dose (Griffiths, 1997). The concentration of residual plasmid DNA in the vector supernatant after Benzonase treatment was below the detection limit of the assay (2.0 pg of DNA per milliliter; Supplementary Table S1). Thus, the calculated concentration of residual plasmid DNA in a single dose of SBVPF LVV after treatment with Benzonase (25 or $50 \mathrm{U} / \mathrm{ml}$; Supplementary Table S1) for $1 \mathrm{hr}$ at room temperature was calculated to be $\leq 0.2 \mathrm{ng} / 100 \mathrm{ml}$. In addition, overnight incubation at room temperature or increasing the temperature to $37^{\circ} \mathrm{C}$ for $1 \mathrm{hr}$ for the Benzonase treatment also resulted in undetectable levels of plasmid DNA (Supplementary Table S1);

A
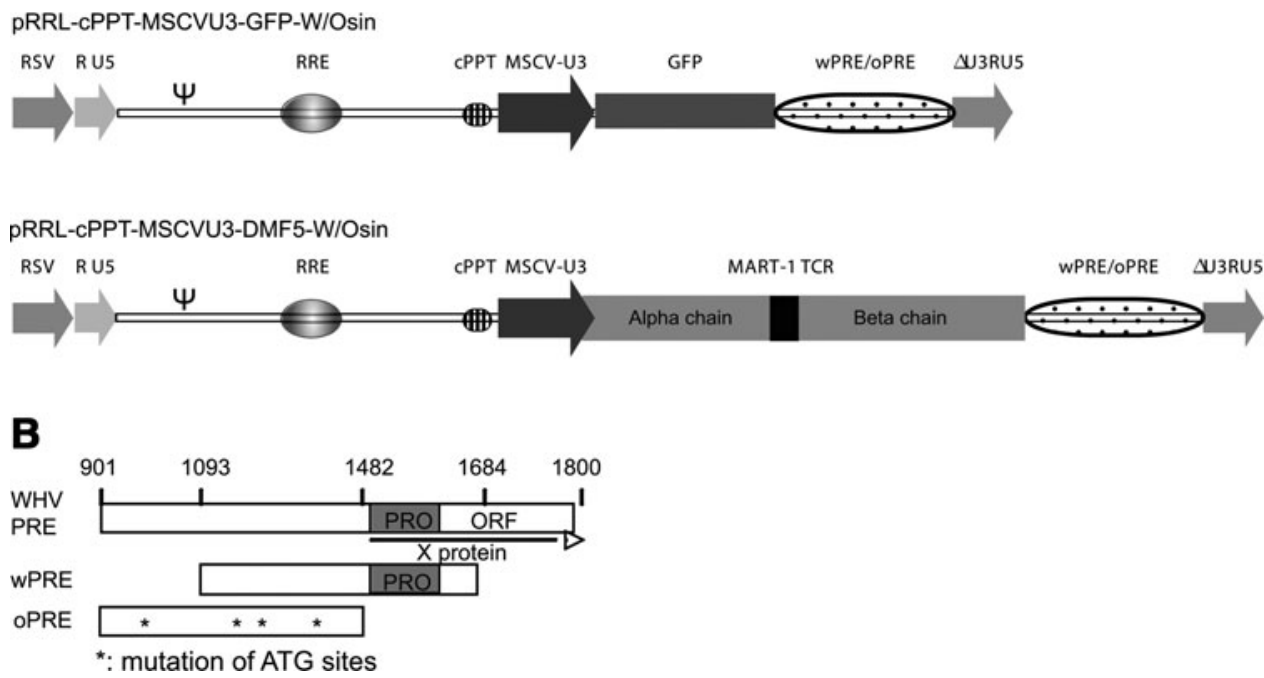

FIG. 1. Design of clinical lentiviral vector backbones. (A) Schematic diagram of pRRL backbones encoding GFP or a codonoptimized MART-1-reactive TCR (DMF5) driven by an internal MSCV U3 promoter. Constructs were made containing wPRE (W) and an optimized version of PRE (oPRE) for comparison. (B) Schematic diagram of woodchuck hepatitis virus posttranslational regulatory element (WHV-PRE), showing the $X$ protein promoter and ORF. wPRE is depicted with the $X$ protein promoter (shaded box) and the majority of the $X$ protein ORF deleted. oPRE is a truncated version of wPRE in which the $X$ protein promoter has been deleted and the ATG start sites (asterisks) for any ORF greater than 25 amino acids mutated to render them nonfunctional. 


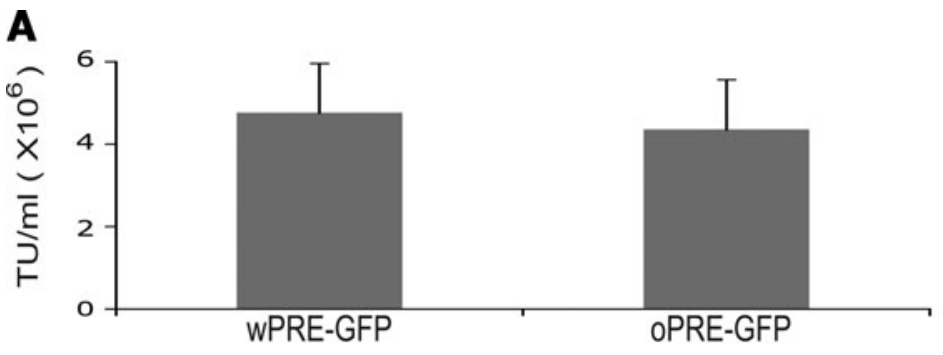

B
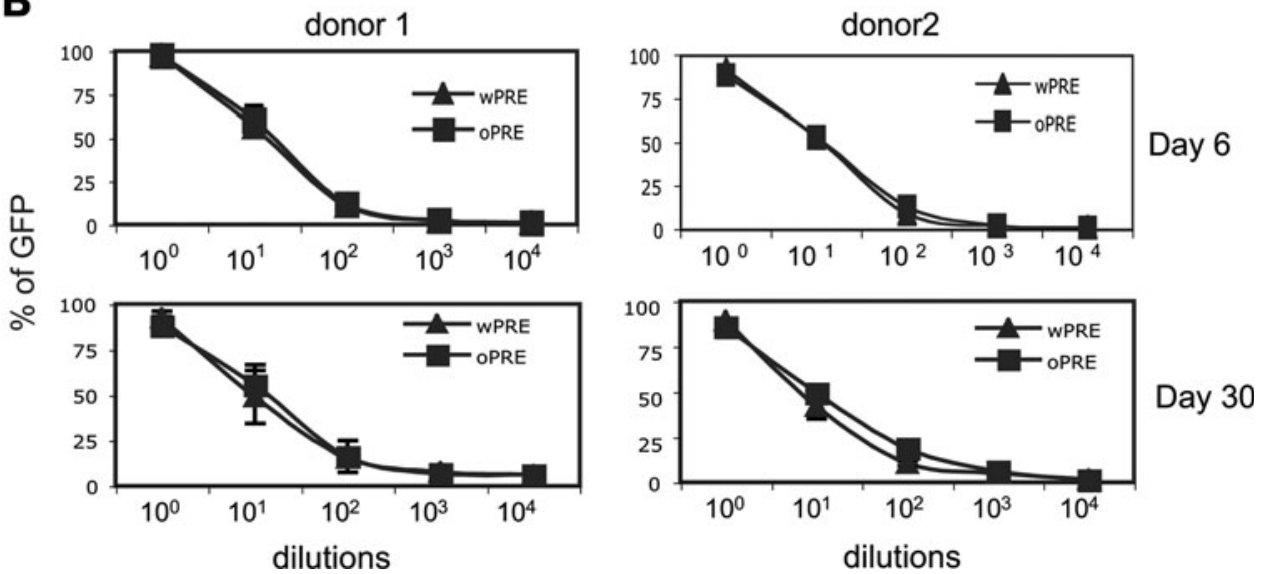

FIG. 2. An optimized PRE sequence (oPRE) can replace wPRE without affecting LVV titer or transgene expression. (A) PBLs from at least three patients were transduced with serial dilutions of pRRL-cPPT-MSCVU3-GFP-Wsin or pRRL-cPPTMSCVU3-GFP-Osin. Eight days posttransduction, GFP-expressing PBLs were detected by FACS. No significant difference in GFP expression was observed between wPRE and oPRE LVV constructs. Titers are presented as transducing units (TU) per milliliter, with error bars representing the standard error of the mean. (B) Dilutions of GFP-encoding vector were used to transduce PBLs and then GFP expression was measured at either day 6 or day 30 by FACS. No significant difference in GFP expression was observed between the wPRE and oPRE LVV constructs at any of the dilutions tested.

however, the LVV titers decreased after these treatments (data not shown).

PBLs transduced with pRRL-cPPT-MSCVU3-GOI-Osin encoding GFP (Fig. 3A) or the coDMF5 TCR (Fig. 3B) resulted in nearly identical levels of GFP and coDMF5 TCR expression, respectively, regardless of whether or not the vector supernatants contained Benzonase $(50 \mathrm{U} / \mathrm{ml})$. In addition, treatment with Benzonase $(50 \mathrm{U} / \mathrm{ml})$ for $1 \mathrm{hr}$ at room temperature did not inhibit the function of coDMF5 TCRtransduced PBLs when cocultured with HLA-matched, MART $-1^{+}$tumor cells (Fig. 3C). Importantly, after PBL transduction in 6-well plates, the PBLs were washed and transferred to flasks for expansion, at which time the amount of residual Benzonase as determined by Benzonase ELISA was below the assay detection limit of $0.2 \mathrm{ng} / \mathrm{ml}$ (Fig. 3D).

\section{A simplified lentivirus production platform}

We compared coDMF5 LVV produced by the simplified SBVPF process with that produced by the IUVPF process, to determine whether there were any differences in transduction efficiency or subsequent function of TCR-transduced PBLs at clinical scale (Fig. 4A). cGMP-quality LVV was produced at both the SBVPF and IUVPF. For this specific production run, the IUVPF process used a concentration step via tangential flow filtration (TFF) to reduce contaminants and the volume of the bulk pooled harvest by 10-fold before Benzonase treatment, followed by diafiltration into the desired excipient. The SBVPF process involved direct treatment of the LVV bulk harvest with Benzonase followed by clarification via modified step filtration (Fig. 4A). Even though the production processes were different for both vector products, the titers of the SBVPF and IUVPF were similar: $5.4( \pm 0.8) \times 10^{7}$ and $5.3( \pm 0.9) \times 10^{7} \mathrm{TU} / \mathrm{ml}$, respectively (Table 1).

Patient PBLs were transduced using a 1:1 dilution starting from $1 \times$ concentrated LVV. For PBLs from the three patients tested, LVV produced by the SBVPF process resulted in slightly increased levels of tetramer-positive cells (Fig. 4B). The higher levels of tetramer-positive cells, after transduction with the SBVPF vector supernatant, did not result in a significant increase in the level of IFN- $\gamma$ secretion when cocultured with $\mathrm{HLA}-\mathrm{A}^{*} 0201^{+} / \mathrm{MART}^{+}{ }^{+}$-matched tumor cells (Fig. 4C). This finding reflects what has been seen when TCR-encoding gammaretroviral vectors are used ex vivo to redirect naive PBLs, and suggests that once a threshold level of antigen-specific TCR is expressed on the surface of the PBLs, additional TCR expression above that threshold does not result in increased IFN- $\gamma$ secretion (Goff et al., 2010). In addition to the removal of residual plasmid DNA, the SBVPF LVV product was tested for carryover of potentially transforming SV40 and adenovirus E1a host cell DNAs from the $293 T$ packaging cells, and was found to be negative for both (Table 1). There was no detection of VSV-G plasmid DNA mobilization in PBLs transduced with the Benzonase-treated LVV product when genomic DNA was evaluated for VSV-G 
A
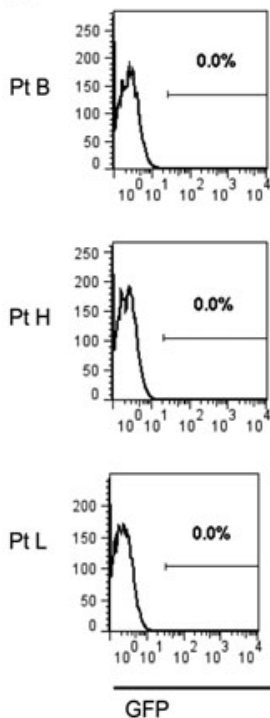

C

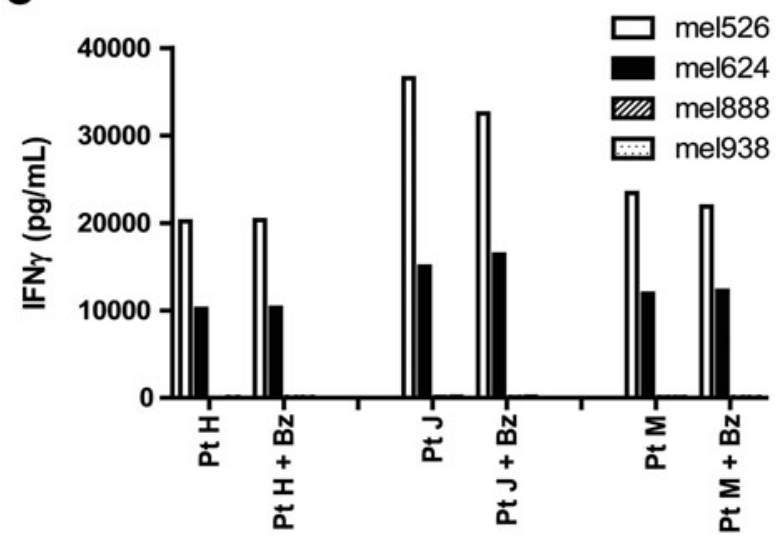

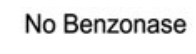
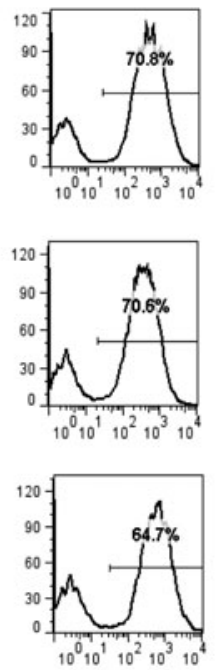
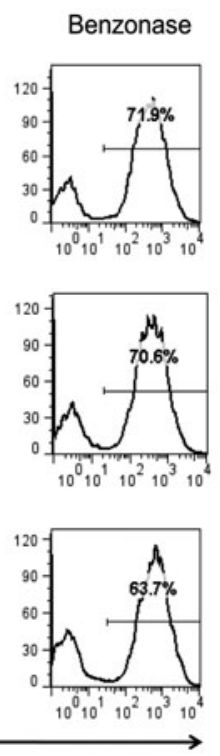

B $\mathrm{PtH}$
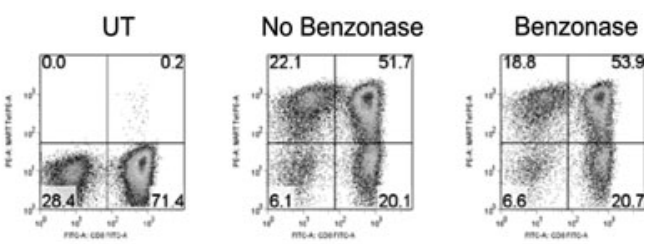

Pt J
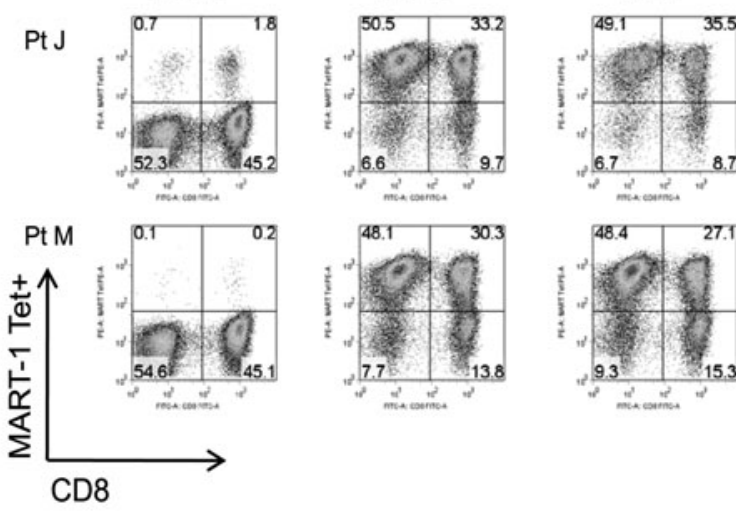

D

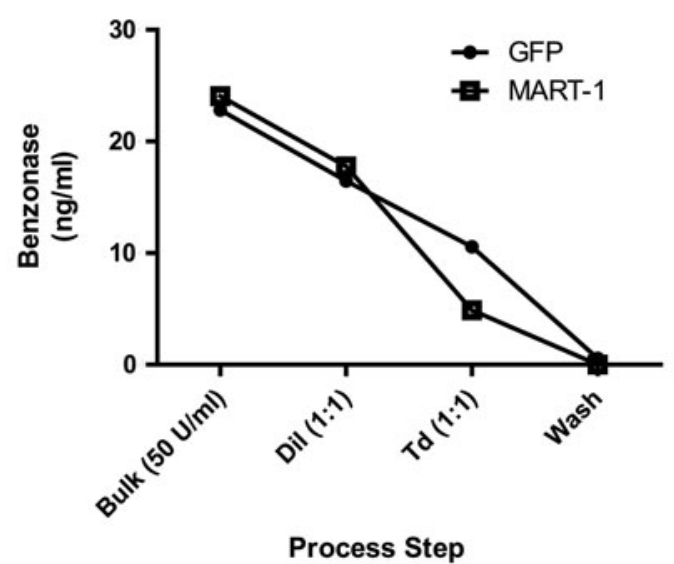

FIG. 3. Treatment of LVV supernatant with Benzonase does not reduce PBL transduction efficiency or function. LVV was treated with Benzonase $(50 \mathrm{U} / \mathrm{ml})$ for $1 \mathrm{hr}$ at room temperature and compared with untreated vector. (A) No significant difference in GFP expression after treatment with Benzonase was observed. (B) No significant difference was observed in DMF5 TCR transduction after treatment with Benzonase. FACS plots for the PBLs of three separate patients are shown. (C) Treated and untreated DMF5 TCR-transduced PBLs were cultured with HLA-A*0201 ${ }^{+}$/MART- ${ }^{+}$(526, open columns; 624, solid columns) and HLA-A2- (888, hatched columns; 938, stippled columns) tumor cell lines. For the PBLs from each of these three patients, there was no significant difference in function as measured by IFN- $\gamma$ release. (D) Benzonase concentration (ng/ $\mathrm{ml}$ ) was measured at various points during the transduction process. Bulk supernatant was treated with Benzonase (50 U/ $\mathrm{ml}$ ), diluted 1:1, adjusted with protamine sulfate to $10 \mu \mathrm{g} / \mathrm{ml}$, and used to transduce PBLs via spinoculation. After a single transduction, PBLs were washed and transferred to an expansion flask, at which point the concentration of Benzonase was below the detection limit of the assay $(<0.2 \mathrm{ng} / \mathrm{ml})$.

sequence by qPCR. In addition, the SBVPF LVV product was negative for replication-competent lentivirus (RCL; Table 1). The IUVPF LVV product was also negative for SV40, adenovirus E1a, and VSV-G envelope DNA mobilization and for RCL (Table 1).

\section{Discussion}

Adoptive cell therapy (ACT) for the treatment of cancer typically involves the transfer into patients of autologous lymphocytes with demonstrated specificity for the tumor, which can result in tumor regression. Naturally occurring tumor-reactive lymphocytes can be isolated and expanded from resected tumor. These tumor-infiltrating lymphocytes (TILs) have been used in the treatment of patients with melanoma, resulting in objective response rates between 49 and $72 \%$ depending on the conditioning regimen (Dudley et al., 2008; Rosenberg et al., 2011). However, for those patients whose tumor cannot generate TILs, an alternative approach exists whereby lymphocytes can be isolated from the peripheral blood of those patients and genetically modified ex vivo to express a tumor-reactive TCR or CAR. Genetically 

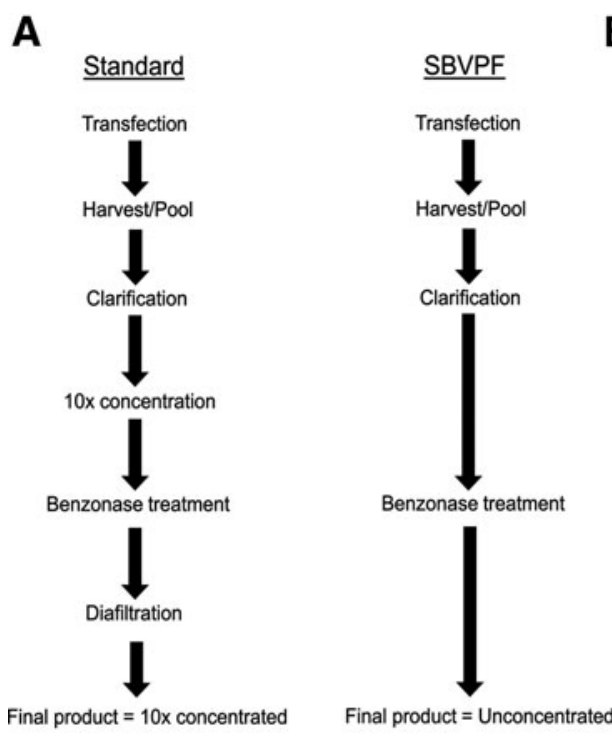

B

Pt. 1

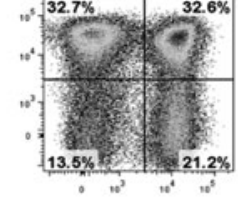

Pt. 2
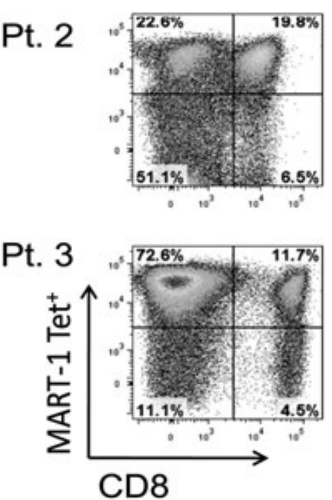

IUVPF
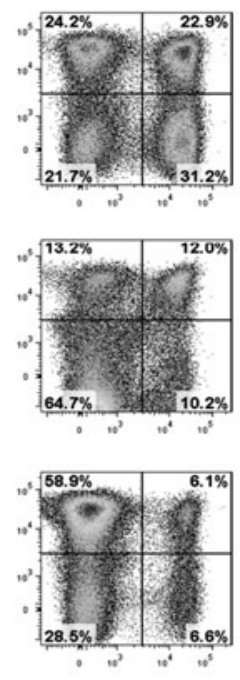

UT
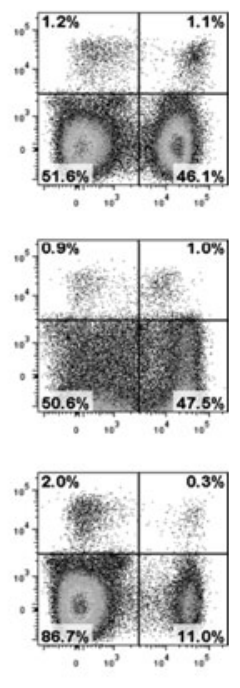

C

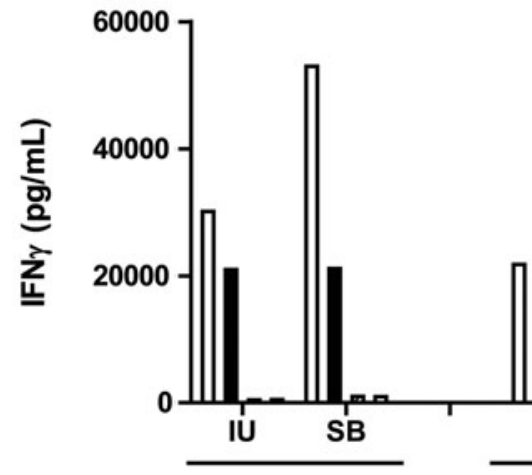

Pt. 1

Pt. 2

Production Process

FIG. 4. Production of clinical-grade LVV using transient transfection and clarification by modified step-filtration. (A) Largescale LVV supernatants were generated at SBVPF and IUVPF as described. 10X concentrated IUVPF LVV supernatant was diluted 10-fold and then both supernatants were diluted 1:1 for PBL transduction. (B) The SBVPF LVV resulted in a higher level of MART-1 tetramer ${ }^{+}$PBL as compared to cGMP vector manufactured at IUVPF. The increased tetramer binding was not significant. (C) PBL were transduced with cGMP g-retroviral vector encoding the DMF5 TCR manufactured at either SBVPF (SB) or IUVPF (IU). TCR-transduced PBL were cultured with HLA-A*0201 ${ }^{+}$, MART- $1^{+}$(526, open bars; 624, solid bars) and HLA-A2 ${ }^{-}$(888, hatched bars; 938, stippled bars) tumor cell lines. For each of the three patients PBL, there was no significant difference in function as measured by IFNg release.

redirected PBLs can mediate tumor regression and may have a significant advantage over TILs in that PBLs can be targeted to a variety of cancers on the basis of the availability of a tumor-specific TCR or CAR (Johnson et al., 2006, 2009; Morgan et al., 2006; Brentjens et al., 2011; Kochenderfer et al., 2011; Parkhurst et al., 2011; Porter et al., 2011; Robbins et al., 2011; Savoldo et al., 2011).

Many institutions performing ACT of mature T lymphocytes have used gammaretroviral vectors as their gene delivery vehicle because of the ease of manufacture and the ability of these vectors to transduce and stably integrate into the lymphocyte genomic DNA, allowing for long-term constitutive expression of a given gene. However, there have been concerns regarding the biosafety of these vectors after transduction of $\mathrm{CD} 34^{+}$stem cells. This concern stems from the fact that several children being treated for X-SCID developed leukemias directly attributed to the gammaretrovirustransduced $\mathrm{CD}^{+} 4^{+}$stem cells administered to correct the common $\gamma$-chain deficiency (Cavazzana-Calvo et al., 2000; Hacein-Bey-Abina et al., 2003). To address biosafety, as well as other concerns such as potential gene silencing and relatively small genetic payloads, many investigators have turned their attention to the development of LVV for gene delivery in $e x$ vivo ACT protocols. However, it should be stated that LVVs, in and of themselves, may not completely solve the problem of insertional mutagenesis, as there has been a single report associating an LVV with clonal dominance in a $\beta$-thalassemia trial (Cavazzana-Calvo et al., 2010).

It is generally believed that LVVs have several advantages over gammaretroviral vectors that make them attractive for 
Table 1. coDMF5 Product Comparison: Standard Versus SBVPF Simplified Process

\begin{tabular}{|c|c|c|}
\hline \multirow[b]{2}{*}{ Residual } & \multicolumn{2}{|c|}{ Production process } \\
\hline & IUVPF & $S B V P F$ \\
\hline Concentration & $10 \times$ & $1 \times$ \\
\hline Titer $\left(\mathrm{TU} / \mathrm{ml}, \times 10^{7}\right)$ & $5.4 \pm 0.8$ & $5.3 \pm 0.9$ \\
\hline Benzonase $(\mathrm{ng} / \mathrm{ml})^{\mathrm{a}}$ & 1.68 & $23.4 \pm 0.6$ \\
\hline Posttransduction ${ }^{\mathrm{b}}$ Benzonase $(\mathrm{ng} / \mathrm{ml})$ & $<0.2$ & $<0.2$ \\
\hline VSV-G env DNA ${ }^{\mathrm{c}}$ & $<10$ & $<10$ \\
\hline Post-Benzonase VSV-G env DNA ${ }^{c, d}$ & $<10$ & $<10$ \\
\hline SV40 DNA ${ }^{\mathrm{c}}$ & $<10$ & $<10$ \\
\hline Adenovirus E1a DNA ${ }^{c}$ & $<10$ & $<10$ \\
\hline RCL & Negative & Negative \\
\hline
\end{tabular}

coDMF5, codon-optimized version of DMF5 $\mathrm{T}$ cell receptor in conjunction with oPRE; IUVPF, Indiana University Vector Production Facility; oPRE, optimized version of wPRE; SBVPF, Surgery Branch Vector Production Facility; RCL, replication-competent retrovirus.

${ }^{\mathrm{a}}$ Benzonase concentration in bulk product, $(\mathrm{ng} / \mathrm{ml})$.

${ }^{b}$ PBLs were transduced two times with $0.5 \times$ lentiviral vector, washed once, and transferred to expansion flasks. Posttransduction samples were pulled from the transfer flask after the wash step. The detection limit of the Benzonase ELISA is $0.2 \mathrm{ng} / \mathrm{ml}$.

'PBLs were transduced with untreated LVV, expanded for 14 days, at which point genomic DNA was extracted and analyzed by qPCR for the presence of VSV-G env, SV40, or adenovirus E1a DNA. The detection limit of the assay is $\geq 10$ copies per $0.2 \mu \mathrm{g}$ of DNA.

${ }^{\mathrm{d}} \mathrm{LVV}$ was treated with Benzonase $(50 \mathrm{U} / \mathrm{ml})$ for $1 \mathrm{hr}$ at room temperature before PBL transduction and then screened by qPCR for VSV-G, SV40, and adenovirus E1a DNAs.

use in gene therapy applications, in addition to the $3^{\prime}$ selfinactivating long terminal repeat (LTR), including a greater packaging capacity (Wiznerowicz and Trono, 2005; Chang and Sadelain, 2007), less susceptibility to gene silencing (Pfeifer et al., 2002; Ikawa et al., 2003), and a decreased propensity for integration into the enhancer/promoter regions of transcriptionally active genes (Montini et al., 2006). LVV backbones have been designed to incorporate the wPRE to stabilize and enhance the accumulation of proviral mRNA (Zufferey et al., 1999; Deglon et al., 2000; Ramezani et al., 2000); however, it has been determined that the sequence encoding the wPRE also encodes the $X$ protein, which may have the potential to be tumorigenic (Bouchard and Schneider, 2004; Schambach et al., 2005).

To improve our LVV design and remove the potentially oncogenic X protein, we replaced the wPRE with the optimized and truncated version, oPRE (Schambach et al., 2005). Inclusion of the oPRE in the pRRL backbone encoding either GFP or the DMF5 TCR had no effect on vector titer or transgene expression as compared with the LVV backbone containing the wPRE (Figs. 1 and 2). Only when the oPRE was used in conjunction with the codon-optimized DMF5 TCR did we see significant functional improvement in TCRtransduced PBLs (Supplementary Fig. S1). It is clear from the data that the oPRE can be used in place of the wPRE in the LVV backbone without any adverse effect on titer or transgene expression. This vector design, in conjunction with codon optimization, can lead to increased TCR expression and function when TCR-transduced PBLs encounter HLA-matched antigen-specific tumor. However, it should be noted that the benefits of codon optimization may be transgene specific and need to be evaluated on a per-construct basis.

One of the major issues limiting the use of LVV for the genetic modification of lymphocytes and other cells ex vivo is the difficulty associated with large-scale LVV manufacturing. In particular, there have been reports regarding a number of packaging cell lines for LVV production (Kafri et al., 1999; Ikeda et al., 2003; Throm et al., 2009; Stewart et al., 2011); however, use of such lines at this time remains challenging. Therefore, most current production efforts rely on large-scale transient transfection of $293 \mathrm{~T}$ cells by a calcium phosphate (Ca-Phos)-based transfection method, primarily because of its low cost. However, this method is extremely sensitive to variations in $\mathrm{pH}$, as well as $\mathrm{Ca}^{2+}$ and phosphate concentrations, which can affect titer and reproducibility between production runs. Transfection reagents such as polyethylenimine (PEI) are being developed for large-scale, serum-free transfection, which should help overcome the challenges associated with transfection using Ca-Phos (Ansorge et al., 2009; Schweizer and Merten, 2010; Segura et al., 2010). An additional challenge hindering the widespread use of LVV is the significant amount of downstream processing required for concentration and purification of the LVV in order to remove contaminating DNA and other impurities. Typical downstream processing for LVV involves clarification of the bulk harvest, concentration, and Benzonase treatment to remove residual plasmid and host cell DNA, followed by additional concentration and/or diafiltration (Fig. 4A). Column chromatography is also routinely being used for vector purification as it allows for clean separation of LVV from other impurities (Schweizer and Merten, 2010; Segura et al., 2010). To simplify the manufacture of LVV for ex vivo ACT protocols, we performed large-scale transient transfection of 293T cells followed by Benzonase treatment $(50 \mathrm{U} / \mathrm{ml})$ and clarification by modified step filtration (Fig. 4A) (Feldman et al., 2011). No additional processing was applied and the bulk product was aliquoted and frozen at $80^{\circ} \mathrm{C}$ until further use. We have shown previously that a concentrated and purified LVV product is not required for efficient ex vivo transduction of PBLs at clinical scale (Yang et al., 2008b). In addition, we and others (Sastry et al., 2004) have shown that Benzonase treatment or the presence of residual Benzonase in the vector supernatant does not inhibit the ability of LVV to efficiently transduce lymphoid cells (Fig. 3B-D). Of note, in our $\mathrm{ACT}$ protocols involving ex vivo PBL transductions by LVV, the level of residual Benzonase was undetectable $(<0.2 \mathrm{ng} / \mathrm{ml})$ after a simple wash step and transfer to the expansion vessel (Fig. 3D). As there is no specific guidance describing the allowable level of residual Benzonase in an LVV product, the higher level of Benzonase $(23.4 \pm 0.6 \mathrm{ng} / \mathrm{ml})$ in the aliquoted and frozen SBVPF product should not pose any additional regulatory or biosafety risk. As stated previously, during the ex vivo transduction and expansion of the mature lymphocytes, the Benzonase is completely removed from the cell product before patient administration.

To get a better sense of how our simplified production platform compared with other cGMP manufacturing processes for gammaretroviral vectors used in phase I/II clinical trials, we generated large-scale transient LVV at the SBVPF and IUVPF as described in Fig. 4A and compared PBL transduction efficiency and biological function, as well as residual contaminants. In the comparison, the SBVPF LVV 
yielded slightly higher levels of TCR transduction. It is possible that the shear forces from the TFF used in the IUVPF production process resulted in disrupted particles or accumulation of an inhibitory factor, such as soluble envelope or extracellular matrix, decreasing the transduction efficiency. Overall, there was no significant difference in the function of PBLs transduced with either vector preparation as measured by IFN- $\gamma$ release after coculture with HLA-matched, MART- ${ }^{+}$ tumor targets (Fig. 4B and C). Importantly, we were unable to detect residual VSV-G plasmid DNA in the LVV supernatant after Benzonase treatment. VSV-G DNA sequence was not detected in genomic DNA from the transduced PBLs, indicating that there was no genomic integration of VSV-G plasmid DNA. We were also unable to detect transfer of potentially transforming host cell DNA from the 293T packaging cell line, including SV40 and adenovirus E1a (Table 1). The final bulk product was tested for replicationcompetent lentivirus and found to be negative. The IUVPF LVV product was also tested and found to be negative for residual VSV-G plasmid DNA, integrating VSV-G plasmid DNA, transforming (SV40 and adenovirus E1a) host cell DNA, and RCL. Thus, it appears as though the SBVPF LVV product is comparable to a cGMP product manufactured for a phase I/II clinical trial at the IUVPF in terms of both safety and efficacy.

The field of gene therapy is vast and involves parenteral administration of genes, as well as the ex vivo modification of cells using both viral and nonviral gene delivery methods. For virus-mediated gene delivery using LVV, one must consider the cell population being transduced (mature lymphocytes or CD34 ${ }^{+}$stem cells), and this should then dictate how the LVV product is manufactured. The IUVPF and others have now moved to incorporating ion-exchange chromatography (IEC) or other purification steps in addition to TFF concentration because purification appears to be important in increasing LVV copy number in transduced CD34 $^{+}$cells (Charrier et al., 2011; Merten et al., 2011). Our data indicate that concentration and purification may not be required when the target cells for transduction are mature lymphocytes. We have presented data herein suggesting that minimally processed LVV can efficiently redirect naive human PBLs ex vivo to become tumor specific. This simplified SBVPF production method can more easily meet the needs of investigator-initiated INDs used in small-scale phase I/II clinical trials for transduction of mature lymphocytes while complying with the current regulatory standards. This simplified production process will decrease the amount of time and effort required for process development, as well as, eliminate the need for concentration and diafiltration, thereby decreasing both the processing time and cost for production for a specific lentiviral vector product. Taken together, the simplified lentiviral production process described herein will make gene-modified lymphocyte ACT clinical trials more accessible to investigators who might otherwise not be able to conduct such studies.

\section{Acknowledgments}

The authors thank Arnold Mixon and Shawn Farid for assistance with flow cytometry. Indiana University is the lentiviral production site for the NHLBI Gene Therapy Re- source Program (HHSN26820074820) and hosts the NCRR National Gene Vector Biorepository (P40 RR024928).

\section{Author Disclosure Statement}

No competing financial interests exist. K.C. is a founder of Rimedion, but there is no financial interest in the work described in this manuscript.

\section{References}

Aiuti, A., Shimon, S., Aker, M., et al. (2002). Correction of ADASCID by stem cell gene therapy combined with nonmyeloablative conditioning. Science 296, 2410-2413.

Ansorge, S., Lanthier, S., Transfiguracion, J., et al. (2009). Development of a scalable process for high-yield lentiviral vector production by transient transfection of HEK293 suspension cultures. J. Gene Med. 11, 868-876.

Blaese, R.M., Culver, K.W., Miller, A.D., et al. (1995). T lymphocyte-directed gene therapy for ADA-SCID: Initial trial results after 4 years. Science $270,475-480$.

Bouchard, M.J., and Schneider, R.J. (2004). The enigmatic X gene of hepatitis B virus. J. Virol. 78, 12725-12734.

Brentjens, R.J., Rivière, I., Park, J.H., et al. (2011). Safety and persistence of adoptively transferred autologous CD19-targeted $\mathrm{T}$ cells in patients with relapsed or chemotherapy refractory B-cell leukemias. Blood 118, 4817-4828.

Cavalieri, S., Cazzaniga, S., Geuna, M., et al. (2003). Human T lymphocytes transduced by lentiviral vectors in the absence of TCR activation maintain an intact immune competence. Blood 102, 497-505.

Cavazzana-Calvo, M., Jacein-Bey, S., de Saint Basile, G., et al. (2000). Gene therapy of human severe combined immunodeficiency (SCID)-X1 disease. Science 288, 669-672.

Cavazzana-Calvo, M., Payen, E., Negre, O., et al. (2010). Transfusion independence and HMGA2 activation after gene therapy of human $\beta$-thalassaemia. Nature 467, 318-322.

Chang, A.H., and Sadelain, M. (2007). The genetic engineering of hematopoietic stem cells: The rise of lentiviral vectors, the conundrum of the LTR, and the promise of lineage-restricted vectors. Mol. Ther. 15, 445-456.

Charrier, S., Ferrand, M., Zerbato, M., et al. (2011). Quantification of lentiviral vector copy numbers in individual hematopoietic colony-forming cells shows vector dose-dependent effects on the frequency and level of transduction. Gene Ther. 18, 479-487.

Deglon, N., Tseng, J.L., Bensadoun, J.-C., et al. (2000). Selfinactivating lentiviral vectors with enhanced transgene expression as potential gene transfer system in Parkinson's disease. Hum. Gene Ther. 11, 179-190.

Dudley, M.E., Yang, J.C., Sherry, R., et al. (2008). Adoptive cell therapy for patients with metastatic melanoma: Evaluation of intensive myeloablative chemoradiation preparative regimens. J. Clin. Oncol. 26, 5233-5239.

Dull, T., Zufferey, R., Kelly, M., et al. (1998). A third-generation lentivirus vector with a conditional packaging system. J. Virol. $72,8463-8471$.

Feldman, S.A., Goff, S.L., Xu, H., et al. (2011). Rapid production of clinical-grade gammaretroviral vectors in expanded surface roller bottles using a "modified" step-filtration process for clearance of packaging cells. Hum. Gene Ther. 22, 107-115.

Gaspar, H.B., Parsley, K.L., Howe, S., et al. (2004). Gene therapy of X-linked severe combined immunodeficiency by use of a pseudotyped gammaretroviral vector. Lancet 364, 2181-2187. 
Goff, S., Johnson, L., Black, M., et al. (2010). Enhanced receptor expression and in vitro effector function of a murine-human hybrid MART-1-reactive T cell receptor following a rapid expansion. Cancer Immunol. Immunother. 59, 1551-1560.

Griffiths, E. (1997). WHO Expert Committee on Biological Standardization: Highlights of the meeting of October 1996. Biologicals 25, 359-362.

Hacein-Bey-Abina, S., von Kalle, C., Schmidt, M., et al. (2003). A serious adverse event after successful gene therapy for $\mathrm{X}$ linked severe combined immunodeficiency. N. Engl. J. Med. $348,255-256$.

Heslop, H.E., Ng, C.Y.C., Li, C., et al. (1996). Long-term restoration of immunity against Epstein-Barr virus infection by adoptive transfer of gene-modified virus-specific $\mathrm{T}$ lymphocytes. Nat. Med. 2, 551-555.

Ikawa, M., Tanaka, M., Kao, W.W.-Y., and Verma, I.M. (2003). Generation of transgenic mice using lentiviral vectors: A novel preclinical assessment of lentiviral vectors for gene therapy. Mol. Ther. 8, 666-673.

Ikeda, Y., Takeuchi, Y., Martin, F., et al. (2003). Continuous hightiter HIV-1 vector production. Nat. Biotechnol. 21, 569-572.

Johnson, L.A., Heemskerk, B., Powell, D.J., Jr., et al. (2006). Gene transfer of tumor-reactive TCR confers both high avidity and tumor reactivity to nonreactive peripheral blood mononuclear cells and tumor-infiltrating lymphocytes. J. Immunol. 177, 6548-6559.

Johnson, L.A., Morgan, R.A., Dudley, M.E., et al. (2009). Gene therapy with human and mouse T-cell receptors mediates cancer regression and targets normal tissues expressing cognate antigen. Blood 114, 535-546.

Jones, S., Peng, P.D., Yang, S., et al. (2009). Lentiviral vector design for optimal $\mathrm{T}$ cell receptor gene expression in the transduction of peripheral blood lymphocytes and tumorinfiltrating lymphocytes. Hum. Gene Ther. 20, 630-640.

Kafri, T., van Praag, H., Ouyang, L., et al. (1999). A packaging cell line for lentivirus vectors. J. Virol. 73, 576-584.

Kalos, M., Levine, B.L., Porter, D.L., et al. (2011). T cells with chimeric antigen receptors have potent antitumor effects and can establish memory in patients with advanced leukemia. Sci. Transl. Med. 3, 95ra73.

Kochenderfer, J.N., Wilson, W.H., Janik, J.E., et al. (2010). Eradication of B-lineage cells and regression of lymphoma in a patient treated with autologous $\mathrm{T}$ cells genetically engineered to recognize CD19. Blood 116, 4099-4102.

Kochenderfer, J.N., Dudley, M.E., Maric, I., et al. (2011). Dramatic regression of chronic lymphocytic leukemia in the first patient treated with donor-derived genetically-engineered anti-CD19-chimeric-antigen-receptor-expressing $\mathrm{T}$ cells after allogeneic hematopoietic stem cell transplantation. Biol. Blood Marrow Transplant. 17, S158-S158.

Lizée, G., Aerts, J.L., Gonzales, M.I., et al. (2003). Real-time quantitative reverse transcriptase-polymerase chain reaction as a method for determining lentiviral vector titers and measuring transgene expression. Hum. Gene Ther. 14, 497-507.

Merten, O.-W., Charrier, S., Laroudie, N., et al. (2011). Largescale manufacture and characterization of a lentiviral vector produced for clinical ex vivo gene therapy application. Hum. Gene Ther. 22, 343-356.

Montini, E., Cesana, D., Schmidt, M., et al. (2006). Hematopoietic stem cell gene transfer in a tumor-prone mouse model uncovers low genotoxicity of lentiviral vector integration. Nat. Biotechnol. 24, 687-696.

Morgan, R.A., Dudley, M.E., Wunderlich, J.R., et al. (2006). Cancer regression in patients after transfer of genetically engineered lymphocytes. Science 314, 126-129.
Parkhurst, M.R., Yang, J.C., Langan, R.C., et al. (2011). T cells targeting carcinoembryonic antigen can mediate regression of metastatic colorectal cancer but induce severe transient colitis. Mol. Ther. 19, 620-626.

Pfeifer, A., Ikawa, M., Dayn, Y., and Verma, I.M. (2002). Transgenesis by lentiviral vectors: Lack of gene silencing in mammalian embryonic stem cells and preimplantation embryos. Proc. Natl. Acad. Sci. U.S.A. 99, 2140-2145.

Porter, D.L., Levine, B.L., Kalos, M., et al. (2011). Chimeric antigen receptor-modified $\mathrm{T}$ cells in chronic lymphoid leukemia. N. Engl. J. Med. 365, 725-733.

Ramezani, A., Hawley, T.S., and Hawley, R.G. (2000). Lentiviral vectors for enhanced gene expression in human hematopoietic cells. Mol. Ther. 2, 458-469.

Robbins, P.F., Morgan, R.A., Feldman, S.A., et al. (2011). Tumor regression in patients with metastatic synovial cell sarcoma and melanoma using genetically engineered lymphocytes reactive with NY-ESO-1. J. Clin. Oncol. 29, 917-924.

Rosenberg, S.A., Yang, J.C., Sherry, R.M., et al. (2011). Durable complete responses in heavily pretreated patients with metastatic melanoma using T-cell transfer immunotherapy. Clin. Cancer Res. 17, 4550-4557.

Sastry, L., Xu, Y., Cooper, R., et al. (2004). Evaluation of plasmid DNA removal from lentiviral vectors by Benzonase treatment. Hum. Gene Ther. 15, 221-226.

Savoldo, B., Ramos, C.A., Liu, E., et al. (2011). CD28 costimulation improves expansion and persistence of chimeric antigen receptor-modified T cells in lymphoma patients. J. Clin. Invest. $121,1822-1826$.

Schambach, A., Bohne, J., Baum, C., et al. (2005). Woodchuck hepatitis virus post-transcriptional regulatory element deleted from $X$ protein and promoter sequences enhances retroviral vector titer and expression. Gene Ther. 13, 641-645.

Schambach, A., Mueller, D., Galla, M., et al. (2006). Overcoming promoter competition in packaging cells improves production of self-inactivating retroviral vectors. Gene Ther. 13, 1524-1533.

Schweizer, M., and Merten, O.-W. (2010). Large-scale production means for the manufacturing of lentiviral vectors. Curr. Gene Ther. 10, 474-486.

Segura, M.M., Garnier, A., Durocher, Y., et al. (2010). New protocol for lentiviral vector mass production. In Lentivirus Gene Engineering Protocols. M. Federico, ed. (Humana Press, New York), pp. 39-52.

Segura, M.M., Kamen, A.A., and Garnier, A. (2011). Overview of Current Scalable Methods for Purification of Viral Vectors. In Viral Vectors for Gene Therapy, O.-W. Merten, and M. AlRubeai, eds. (Humana Press), pp. 89-116.

Shaw, K.L., and Kohn, D.B. (2011). A tale of two SCIDs. Sci. Transl. Med. 3, 97ps36.

Stewart, H.J., Fong-Wong, L., Strickland, I., et al. (2011). A stable producer cell line for the manufacture of a lentiviral vector for gene therapy of Parkinson's disease. Hum. Gene Ther. 22, 357-369.

Throm, R.E., Ouma, A.A., Zhou, S., et al. (2009). Efficient construction of producer cell lines for a SIN lentiviral vector for SCID-X1 gene therapy by concatemeric array transfection. Blood 113, 5104-5110.

Topalian, S., Solomon, D., and Rosenberg, S. (1989). Tumorspecific cytolysis by lymphocytes infiltrating human melanomas. J. Immunol. 142, 3714-3725.

Walter, E.A., Greenberg, P.D., Gilbert, M.J., et al. (1995). Reconstitution of cellular immunity against cytomegalovirus in recipients of allogeneic bone marrow by transfer of T-cell clones from the donor. N. Engl. J. Med. 333, 1038-1044. 
Wiznerowicz, M., and Trono, D. (2005). Harnessing HIV for therapy: Basic research and biotechnology. Trends Biotechnol. 23, 42-47.

Woods, N.-B., Bottero, V., Schmidt, M., et al. (2006). Gene therapy: Therapeutic gene causing lymphoma. Nature 440, 1123-1123.

Wu, X., Li, Y., Crise, B., and Burgess, S.M. (2003). Transcription start regions in the human genome are favored targets for MLV integration. Science 300, 1749-1751.

Yang, S., Cohen, C.J., Peng, P.D., et al. (2008a). Development of optimal bicistronic lentiviral vectors facilitates high-level TCR gene expression and robust tumor cell recognition. Gene Ther. $15,1411-1423$.

Yang, S., Rosenberg, S.A., and Morgan, R.A. (2008b). Clinicalscale lentiviral vector transduction of PBL for TCR gene therapy and potential for expression in less-differentiated cells. J. Immunother. 31, 830-839.

Zufferey, R., Donello, J.E., Trono, D., and Hope, T.J. (1999). Woodchuck hepatitis virus posttranscriptional regulatory element enhances expression of transgenes delivered by retroviral vectors. J. Virol. 73, 2886-2892.

Address correspondence to: Dr. Steven A. Feldman Surgery Branch National Cancer Institute National Institutes of Health Bethesda, MD 20892

E-mail: feldmanst@mail.nih.gov

Received for publication November 14, 2011; accepted after revision March 1, 2012.

Published online: March 19, 2012. 